\title{
Individualised Surgical Treatment of Patients with an Adenocarcinoma of the Distal Oesophagus or Gastro-Oesophageal Junction
}

\author{
J.B.F. Hulscher J.J.B. van Lanschot \\ Department of Surgery, Academic Medical Centre, University of Amsterdam, Amsterdam, The Netherlands
}

\section{Key Words}

Adenocarcinoma - Distal oesophagus - Gastro-

oesophageal junction - Oesophageal adenocarcinoma •

Oesophageal surgery

\begin{abstract}
In this review we discuss the different strategies to improve surgical outcomes after potentially curative resection for oesophageal adenocarcinoma. For tumours of the distal oesophagus, there is a $17 \%$ survival benefit after transthoracic resection with two-field lymph node dissection when compared with transhiatal resection. This survival benefit is absent for tumours of the gastrooesophageal junction or gastric cardia. These patients should, in the absence of tumour-positive lymph nodes at or proximal to the carina, undergo a transhiatal resection to minimise peri-operative complications. New developments include endoscopic resection or minimally invasive oesophagectomy, but these therapies should still be considered experimental.
\end{abstract}

Copyright $(2005$ S. Karger AG, Basel

\section{Introduction}

Optimising the treatment of oesophageal cancer patients consists of different strategies: early diagnosis, correct patient selection, optimal operative resection and peri-operative care, and possibly the application of (neo-)adjuvant chemo-radiation therapy. Oesophageal cancer surgery is highly complex, and oesophageal carcinoma warrants a multidisciplinary approach. Gastroenterologists, surgeons, pathologists, anaesthetists, radiologists and other (para)medical staff should collaborate closely to optimise care for these patients. Whereas surgery is generally considered to offer the best chance for cure in the absence of local irresectability and/or distant metastases, opinions on how to improve survival rates by surgery are conflicting. In this review, we discuss the different strategies to improve surgical outcome, focussing on the extent of resection.

\section{Extended vs. Limited Resection}

In major cancer surgery, there are two main strategies to improve surgical outcome. One strategy aims at decreasing early morbidity and mortality by performing a relatively limited resection. Opponents of this strategy claim that the performance of a more extended resection might improve staging of the tumour, that it might de-

J.J.B. van Lanschot

Department of Surgery, Academic Medical Centre

University of Amsterdam, Meibergdreef 9

NL-1105 AZ Amsterdam (The Netherlands)

Tel. +31 20566 2766, Fax +31 20691 4858, E-Mail J.J.vanlanschot@amc.uva.nl 
crease the incidence of loco-regional recurrence and that it might improve long-term survival.

For oesophageal tumours a limited resection implies the performance of a cervico-abdominal (transhiatal) oesophagectomy without formal lymphadenectomy $[1,2]$. The avoidance of a thoracotomy should lead to a lower incidence of (pulmonary) complications, and possibly a decrease in early mortality without influencing long-term results. An extended resection implies a combined (cervico-)thoraco-abdominal resection, with wide excision of the tumour and peri-tumoural tissues, and an extended lymph node dissection of the posterior mediastinum and the upper abdomen (transthoracic oesophagectomy with two-field lymphadenectomy) [3, 4]. In some (mostly Japanese) centres, a third field is added in the form of cervical lymphadenectomy $[5,6]$. Besides offering the possibility of an extended lymph node dissection, performing a thoracotomy might enable the surgeon to perform a more radical local resection, thereby increasing the number of radical $\mathrm{R} 0$ resections, one of the main prognostic factors in oesophageal cancer surgery.

Staging is improved in one quarter of the patients after extended resection [7]. This is mainly due to the finding of tumour-positive lymph nodes near the coeliac axis, which are considered distant metastases for distal oesophageal carcinoma. This effect of a more extended resection on staging has also been described for other gastrointestinal tumours, such as gastric carcinoma [8]. The subsequent stage migration might influence the comparison of different resection forms on a stage-by-stage basis, but does not influence comparison of overall survival rates in different series.

Two recent meta-analyses of the English literature of the last decade showed comparable 5-year survival rates (around 25\%) after transthoracic resection and after transhiatal resection $[9,10]$. However, these meta-analyses could be criticised as comparing surgical procedures rather than the extent of resection, and at the time of publication only 3 small, underpowered, randomised studies had been published comparing the different resection forms. These 3 randomised studies combined included a total of 138 patients. Main surgical outcomes (early morbidity and mortality, long-term survival) were not significantly different between the transhiatal and transthoracic groups [11-13].

We have recently published a randomised study comparing transthoracic resection and transhiatal resection for adenocarcinoma of the distal oesophagus or gastric cardia [14].

Optimising Surgical Treatment for

Oesophageal Adenocarcinoma
Between April 1994 and February 2000, 220 patients with adenocarcinoma of the mid/distal oesophagus or gastric cardia involving the distal oesophagus were randomised for transhiatal oesophagectomy or transthoracic oesophagectomy with two-field lymphadenectomy. The main endpoints were overall survival and disease-free survival. Early morbidity and mortality, quality-adjusted life years and cost-effectiveness were also determined.

One hundred and six patients were randomised for transhiatal oesophagectomy, 114 for transthoracic oesophagectomy. Demographic and tumour characteristics were comparable. Peri-operative morbidity was higher after transthoracic oesophagectomy, leading to a significantly prolonged ventilation time (median 1 vs. 2 days), ICU/ MCU stay (median 2 vs. 6 days) and hospital stay (median 15 vs. 19 days). Hospital mortality was $2 \%$ after transhiatal resection and $4 \%$ after transthoracic resection $(p=0.45)$.

The radicality of surgery $(\mathrm{R} 0$ resections in $72 \%$ after transhiatal resection vs. $71 \%$ after transthoracic resection) and pTNM stages were comparable. By the end of follow-up 142 patients had died, 74 (69\%) after transhiatal resection and $68(60 \%)$ after transthoracic resection $(p=0.12)$. Although the difference in survival was not statistically significant, there was a trend towards a survival benefit of the extended approach at 5 years: diseasefree survival was 27 vs. $39 \%$, while overall survival was 29 vs. $39 \%$. Costs of treatment were EUR 23,809 and 37,099 , respectively, while there was no prolonged difference in quality of life between both groups.

The long-term benefit of transthoracic resection could be attributed to patients with a distal oesophageal carcinoma (Siewert type I), in which the estimated 5-year survival benefit for transthoracic resection was $17 \%(95 \%$ confidence interval of the difference -3 to $37 \%$ ) (fig. 1). The survival benefit of a transthoracic resection for patients with a carcinoma of the cardia/gastro-oesophageal junction (Siewert type II) was only 1\% (fig. 2). In this subgroup analysis, oesophageal carcinoma was defined (in the resection specimen) as tumour in the oesophagus, while cardia or junction tumours were defined as the bulk of the tumour in the gastric cardia or at the gastrooesophageal junction. Based on this subgroup analysis, we now prefer the transhiatal route for patients with a cardia/junction carcinoma, unless tumour-positive lymph nodes have been identified at or proximal to the carina during pre-operative work-up, because these lymph nodes cannot be removed via the transhiatal route. In those cases we perform a transthoracic resection with two-field lymphadenectomy, just as we do for patients with a tumour of the oesophagus. 


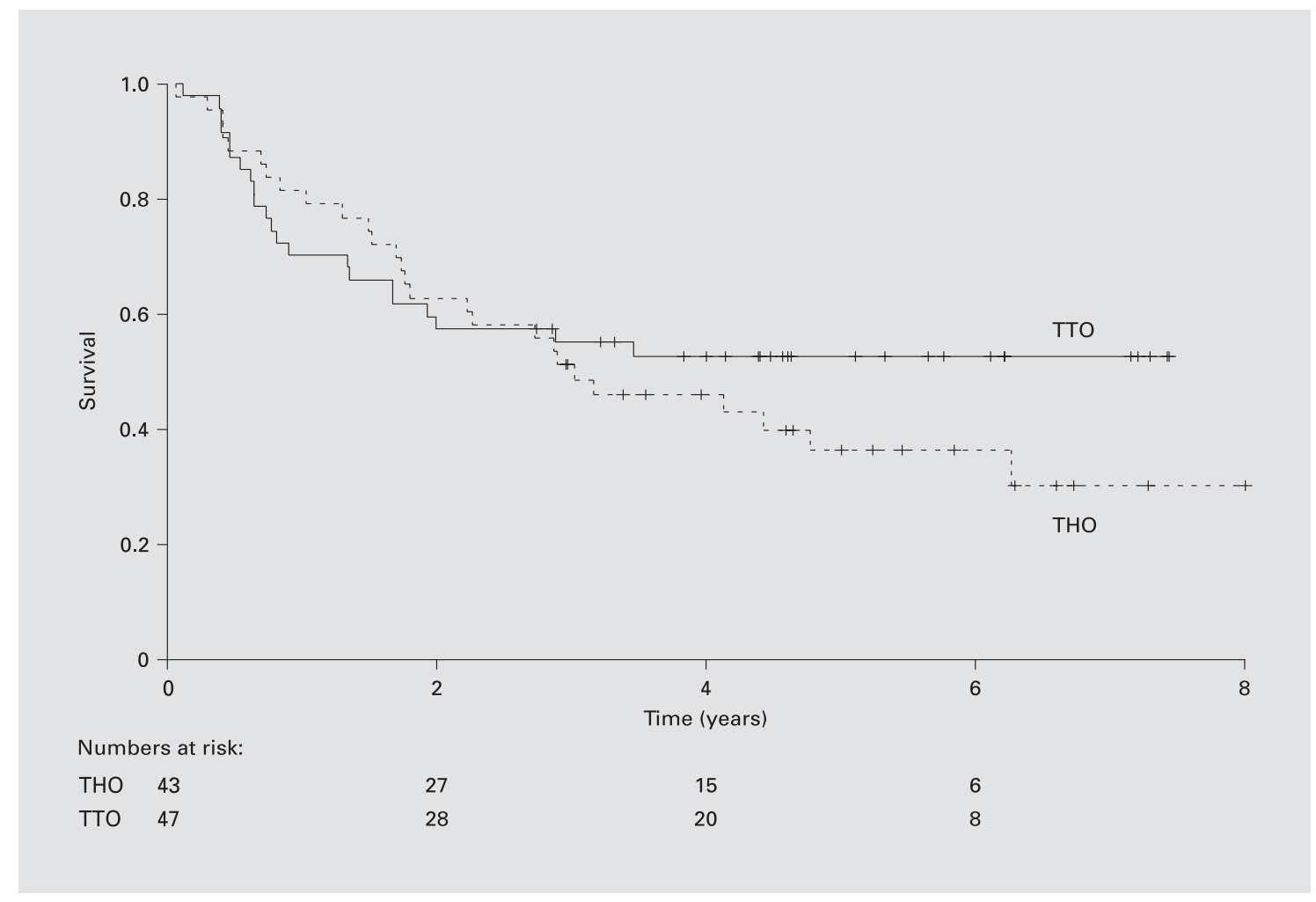

Fig. 1. Survival curves of patients undergoing transhiatal or transthoracic resection for adenocarcinoma of the distal oesophagus (Siewert 1). THO = Transhiatal oesophagectomy; TTO = transthoracic oesophagectomy.

\section{Possible Future Strategies}

Some, mostly Japanese, surgeons argue that an extended two-field lymphadenectomy is not even sufficient due to the high risk of cervical lymph node metastases, and one should therefore perform a three-field lymphadenectomy. It should be noted that there is considerably more morbidity after a two-field lymphadenectomy when compared with a limited transhiatal resection. Also, cervical recurrence is a rare clinical event in our (Western) population, occurring in only $6 \%$ of our patients after a transhiatal resection [15]. Adding a formal cervical dissection would only add to the already increased morbidity, and until large randomised trials demonstrate a clear survival benefit (also taking into consideration quality of life), a three-field lymphadenectomy is, in our opinion, not warranted.

Another option would be to minimise the limited transhiatal procedure even further to diminish post-operative morbidity and mortality. First reports of minimally invasive oesophagectomy are emerging, with decreased morbidity and mortality, less post-operative pain, a shorter hospital stay and earlier return to work as the alleged advantages. Luketich et al. [16] recently published a series of 222 patients in whom minimally invasive oesophagectomy (MIO) was performed. MIO was successfully completed in $93 \%$ of the patients, with an operative mortality rate of $1.4 \%$. Hospital stay (7 days) was shorter than mentioned in most open series, although some reports mention a hospital stay of 1 week after open transhiatal oesophagectomy [1]. Stage-specific survival was similar to open series, suggesting that in experienced hands MIO is both feasible and oncologically safe. Of course, this promising new technology should preferably be tested in randomised studies comparing optimal open surgery with optimal MIO.

Small mucosal tumours (T1a) can now be removed endoscopically with endoscopic mucosal resection (EMR) or ablative techniques such as photodynamic therapy, thus avoiding the morbidity and mortality associated with oesophagectomy. The main problem with these new treatment modalities is the early longitudinal lymphatic dissemination in patients with oesophageal cancer. While 


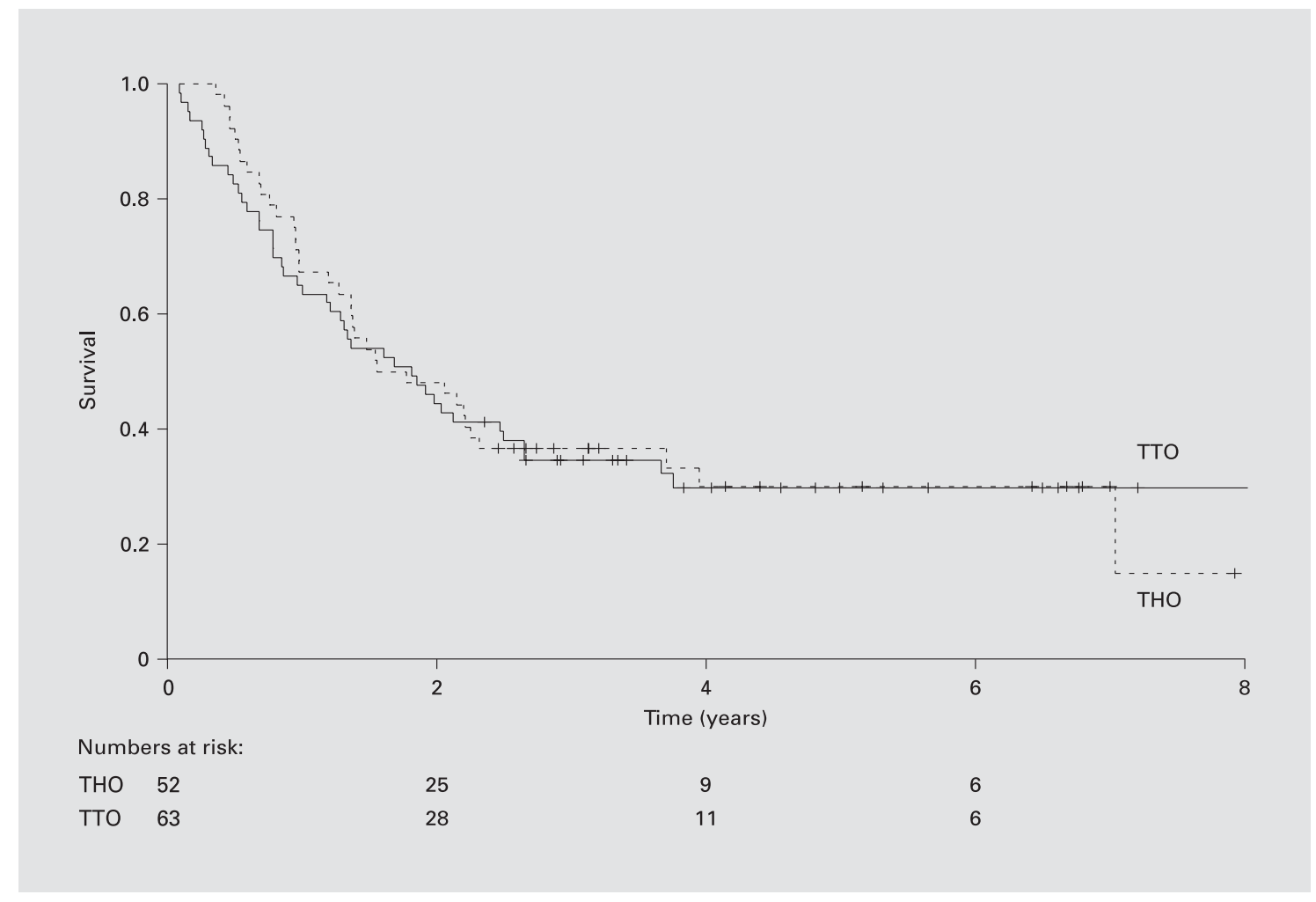

Fig. 2. Survival curves of patients undergoing transhiatal or transthoracic resection for adenocarcinoma of the gastro-oesophageal junction or gastric cardia (Siewert 2). THO = Transhiatal oesophagectomy; TTO = transthoracic oesophagectomy.

lymph node metastases are almost never found in tumours confined to the mucosa ( $\mathrm{T} 1 \mathrm{a})$, lymph node metastases are present in up to $20-30 \%$ of patients with tumours extending into the submucosa (T1b) $[17,18]$. A thorough work-up, consisting of accurate endosonography, external sonography (both when necessary combined with fine-needle aspiration) and CT scanning is therefore essential for deciding upon the optimal treatment strategy. This diagnostic information can be used to identify patients probably having T1aN0 tumours, who are candidates for local endoscopic therapies. One option currently in use is to perform EMR when a T1 tumour is suspected by endosonography. In the EMR specimen the extent of infiltration can subsequently be assessed. When the early lesion is confined to the mucosa, the EMR can be considered as curative, while in case of submucosal infiltration the patient will subsequently have to undergo surgical resection of the oesophagus [19]. These new endoscopic strategies are still to be considered experimental, as no long-term results are available yet.
Considering the fact that oesophagectomy is a major surgical procedure, with significant morbidity and mortality, surgeons should always be aware of their own performance. As for most major types of surgery, there seems to be an inverse relation between hospital volume and hospital mortality for oesophagectomy. In a recent series from the United States, mortality rates from oesophagectomy ranged from $8 \%$ in high-volume centres to as much as $23 \%$ in low-volume centres [20]. These results have been confirmed by a Dutch study in which mortality in centres performing over 50 oesophagectomies per year was 5 vs. $12 \%$ in hospitals performing less than 10 oesophagectomies per year [21]. Hospital volume reflects the experience of the entire hospital, including surgical, anaesthesiologic, medical, and nursing staff. It is probably not the single surgeon who makes the difference, but the multidisciplinary team by which the diagnostic workup and subsequent therapy are performed.

Optimising the treatment of oesophageal cancer patients consists of different strategies: early diagnosis, optimal patient selection, optimal peri-operative care, and 
possibly the application of (neo-)adjuvant chemoradiation therapy. Oesophageal cancer surgery is highly complex, and oesophageal carcinoma warrants a multidisciplinary approach. Gastroenterologists, surgeons, pathologists, anaesthetists, radiologists and other (para)medical staff should collaborate closely to optimise care for these patients.

\section{Conclusion}

Based on the presently available data, the treatment for carcinoma of the oesophagus becomes more and more tailor-made. Treatment of oesophageal carcinoma should preferably take place in hospitals with a wide experience with this disease. Small mucosal tumours can be cured via endoscopic techniques. If the tumour invades the submucosa, a formal oesophageal resection should be performed. A carcinoma of the mid/distal oesophagus in a physically fit patient should preferably be removed via a transthoracic resection with two-field lymph node dissection, while a tumour of the gastro-oesophageal junction or the gastric cardia involving the distal oesophagus should be removed via a transhiatal resection. However, when in case of a cardiac/junction tumour intrathoracic lymph nodes at or above the carina (which cannot be removed transhiatally) are found to be tumour-positive, a transthoracic resection with two-field lymphadenectomy is recommended.

\section{References}

1 Orringer MB, Marshall B, Iannettoni MD: Transhiatal esophagectomy: Clinical experience and refinements. Ann Surg 1999;230: 392-403.

$\checkmark 2$ Gupta NM: Oesophagectomy without thoracotomy: First 250 patients. Eur J Surg 1996; 162:455-461.

>3 Hagen JA, DeMeester SR, Peters JH, Chandrasoma $\mathrm{P}$, DeMeester TR: Curative resection for esophageal adenocarcinoma. Ann Surg 2001;234:520-531.

-4 Altorki N, Skinner D: Should en bloc esophagectomy be the standard of care for esophageal carcinoma? Ann Surg 2001;234:581-587.

-5 Matsubara T, Ueda M, Nagao N, Takahashi T, Nakajima T, Nishi M: Cervicothoracic approach for total meso-esophageal dissection in cancer of the thoracic esophagus. J Am Coll Surg 1998; 187:238-245.

-6 Altorki NK, Skinner DB: Occult cervical nodal metastasis in esophageal cancer: Preliminary results of three-field lymphadenectomy. J Thorac Cardiovasc Surg 1997;113:540-544.

7 Hulscher JBF, Van Sandick JW, Offerhaus GJA, Tilanus HW, Obertop H, Van Lanschot JJB: A prospective analysis of the diagnostic yield of extended en bloc resection for adenocarcinoma of the oesophagus or gastric cardia. Br J Surg 2001;88:715-719.
8 Bunt AMG, Hermans J, Smit VTHBM, et al: Surgical/pathological-stage migration confounds comparisons of gastric cancer survival rates between Japan and western countries. J Clin Oncol 1995;13:19-25.

-9 Rindani R, Martin CJ, Cox MR: Transhiatal versus Ivor-Lewis oesophagectomy: Is there a difference? Aust NZ J Surg 1999;69:187-194.

10 Hulscher JBF, Tijssen JGP, Obertop H, Van Lanschot JJB: Transthoracic versus transhiatal resection for carcinoma of the esophagus: A meta-analysis. Ann Thorac Surg 2001;72:306313.

11 Goldminc M, Maddern G, LePrise E, Meunier B, Campion JP, Launois B: Oesophagectomy by transhiatal approach or thoracotomy: A prospective randomised trial. Br J Surg 1993;80: 367-370.

12 Chu KM, Law SY, Fok M, Wong J: A prospective randomized comparison of transhiatal and transthoracic resection for lower-third oesophageal carcinoma. Am J Surg 1997;174:320 324.

13 Jacobi CA, Zieren HU, Müller M, Pichlmaier $\mathrm{H}$ : Surgical therapy of oesophageal carcinoma: The influence of surgical approach and oesophageal resection on cardiopulmonary function. Eur J Cardiothorac Surg 1997;11:32-37.

14 Hulscher JBF, Van Sandick JW, De Boer AGEM, et al: Extended transthoracic resection compared with limited transhiatal resection for adenocarcinoma of the esophagus. N Engl J Med 2002;347:1662-1669.
15 Hulscher JBF, Van Sandick JW, Tijssen JGP, Obertop H, Van Lanschot JJB: The recurrence pattern of esophageal carcinoma after transhiatal resection. J Am Coll Surg 2000;191:143148.

16 Luketich JD, Alvelo-Rivera M, Buenaventura PO, et al: Minimally invasive esophagectomy. Ann Surg 2003;238:486-494.

17 Nigro JJ, Hagen JA, DeMeester TR, et al: Occult esophageal adenocarcinoma: Extent of disease and implications for effective therapy. Ann Surg 1999;230:433-440.

18 Van Sandick JW, Van Lanschot JJB, Ten Kate FJW, et al: Pathology of early invasive adenocarcinoma of the esophagus or esophagogastric junction: Implications for therapeutic decision making. Cancer 2000;88:2429-2437.

19 May A, Gossner L, Behrens A, et al: A prospective randomized trial of two different endoscopic resection techniques for early stage cancer of the esophagus. Gastrointest Endosc 2003; 58:167-175.

20 Birkmeyer JD, Siewers AE, Finlayson EVA, et al: Hospital volume and surgical mortality in the United States. N Engl J Med 2002;346: 1128-1137.

-21 Van Lanschot JJB, Hulscher JBF, Buskens CJ, Tilanus HW, Ten Kate FJW, Obertop H: Hospital volume and hospital mortality for esophagectomy. Cancer 2001;91:1574-1578. 